\title{
2-Substituted Benzimidazole Synthesis in Dry Medium Mediated by Pyridine $\mathrm{N}$-oxide
}

\author{
GEORGE BRATULESCU* \\ University of Craiova, Faculty of Sciences, Department of Chemistry, 13 A. I. Cuza, 200585, Craiova, Romania
}

\begin{abstract}
Substituted benzimidazoles were obtained from o-phenylenediamine and organic halides in solvent-free medium. The procedure involves pyridine $\mathrm{N}$-oxide as mild oxidizing agent. The benzimidazoles were prepared without the separation of the intermediates. The absence of catalysts and good yields are important benefits of the method.
\end{abstract}

Keywords: Benzimidazoles, halogenated compounds, oxidation, multicomponent reactions

Benzimidazole is a bicyclic compound containing the benzene ring and the imidazole nucleus, both fused. The heterocyclic moiety of benzimidazole derivatives is important in pharmaceutical and medicinal chemistry, many compounds show a high biological activity [1].

Benzimidazole derivatives exhibit antiprotozoal activity against Giardia intestinalis, Trichomonas vaginalis and Entamoeba histolytica [2], antimicrobial activity against a range of bacterial and fungal strains [3-6], and antiviral therapeutic effects [7-9]. Some benzimidazoles are used as anti-inflammatory agents with antioxidant effect [10, 11], antiulcer drugs [12] and anticancer compounds [13, 15].

The complexes of transition metal salts with benzimidazole derivatives reveal pharmacological properties. Thus, the complexes of copper (II) [16], ruthenium (II) [17], cobalt (II) [18], platinum (II) and palladium (II) [19] with benzimidazole derivatives shows high cytotoxic activity.

Historically, the first synthesis of a benzimidazole derivative, 2,5(or 2,6)-dimethylbenzimidazole, was accomplished in 1872 by F. Hoebrecker from 2-nitro-4methylacetanilide and tin in aqueous hydrochloric acid medium $[20,21]$.

It should also be noted that several methods have been developed for synthesis of 2-substituted benzimidazole. Condensation reaction between o-phenylenediamines and carboxylic acids, nitriles, acyl chlorides, orthoesters and imidates occurs at high temperatures in strong acidic medium [22-24]. Condensation reaction of 1,2phenylenediamines and aldehydes followed by a dehydrogenation require different oxidants to afford 2substituted benzimidazoles as well [25-30]. 2-Substituted benzimidazoles can also be prepared from 2-haloanilides via intramolecular cyclization catalyzed by transition metals as iridium [31], iron [32], copper [33], and zirconium [34].

Unconventional methods involving microwaves $[35,36]$ and ultrasound [37] as activation sources have also been used for 2-substituted benzimidazole synthesis.

Although all of these methods are widely used for the synthesis of 2-substituted benzimidazoles, they have

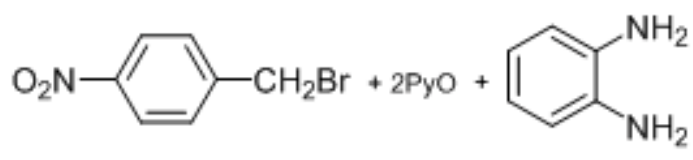

important inconvenient such as long reaction time, low yields, expensive catalysts, tedious protocols, hazardous solvents, and in some cases, sever reaction conditions are imperious.

Earlier, we obtained benzimidazole derivatives through a domino reaction [38, 39]. Following this subject, we obtained various aromatic and aliphatic 2-substituted benzimidazoles starting from o-phenylenediamine, halogenated compounds and pyridine- $N$-oxide.

\section{Experimental part}

Reagents, equipment and methods

Orthophenylenediamines, pyridine- $\mathrm{N}$-oxide and organobromides are commercial substances. Synthesized products were identified using TLC (petroleum etherEtOAC), IR spectra and elemental analysis. IR / FT spectra were recorded with an ALPHA FTIR / ATR spectrometer produced by Bruker Optics $\mathrm{GmbH}$. Elemental analyses were accomplished with a Carlo Erba model 1106 elemental analyzer fabricated by Carlo Erba SpA. The melting points were measured on a Gallenkamp digital melting point apparatus manufactured by Sanyo Electric Co.

\section{General procedure of synthesis}

In a round bottom flask fitted with a reflux bulb condenser were introduced primary organobromides $(5 \mathrm{mmol})$, pyridine- $\mathrm{N}$-oxide ( $12.5 \mathrm{mmol}$ ) and o-phenylenediamine derivative $(5 \mathrm{mmol})$. The mixture was placed in an oil bath on a magnetic stirrer hot plate at required temperature and heated to selected temperature for required time. At the end of the reaction, the organic mixture was washed with dilute $\mathrm{NaOH}$ and filtered off. The resulting precipitate was recrystallized from $\mathrm{EtOH}-\mathrm{H}_{2} \mathrm{O}$ to give the desired 2substituted benzimidazole products (table 2). Melting points of the benzimidazole derivatives are identical with the literature melting points of the same compounds.

\section{Results and discussions}

First, we explored the reaction of obtaining 2-(4nitrophenyl)- $1 \mathrm{H}$-benzimidazole (table 1 ). The reaction occurs as follows:

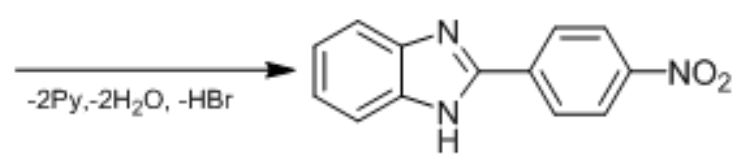

* email: georgebratulescu@yahoo.com 


\begin{tabular}{|c|c|c|c|c|c|c|}
\hline \multirow[t]{2}{*}{ Entry } & \multicolumn{3}{|l|}{ Molar ratio } & \multirow{2}{*}{$\begin{array}{l}\text { Time } \\
{[\mathrm{min}]}\end{array}$} & \multirow{2}{*}{$\begin{array}{l}\mathrm{T} \\
{\left[{ }^{\circ} \mathrm{C}\right]}\end{array}$} & \multirow{2}{*}{$\begin{array}{l}\text { Yield } \\
{[\%]}\end{array}$} \\
\hline & $\mathrm{p}-\mathrm{O}_{2} \mathrm{NC}_{6} \mathrm{H}_{4} \mathrm{CH}_{2} \mathrm{Br}$ & PyO & $1,2-\left(\mathrm{NH}_{2}\right)_{2} \mathrm{C}_{6} \mathrm{H}_{4}$ & & & \\
\hline 1 & 1 & 1 & 1 & 150 & 100 & 42 \\
\hline 2 & 1 & 1 & 2 & 140 & 110 & 83 \\
\hline 3 & 1 & 1.5 & 1 & 120 & 105 & 67 \\
\hline 4 & 1 & 2.5 & 1 & 120 & 110 & 90 \\
\hline 5 & 1.5 & 1 & 1 & 135 & 110 & 69 \\
\hline 6 & 1 & 2 & 1 & 120 & 105 & 80 \\
\hline 7 & 1 & 2.5 & 1 & 135 & 100 & 75 \\
\hline 8 & 1 & 2.5 & 1 & 130 & 105 & 90 \\
\hline 9 & 1 & 2.5 & 1.5 & 120 & 105 & 85 \\
\hline 10 & 1.5 & 2.5 & 1 & 130 & 110 & 73 \\
\hline 11 & 1 & 2.5 & 2 & 140 & 110 & 89 \\
\hline
\end{tabular}

Table 1

THE INFLUENCE OF REACTION CONDITIONS FOR 2-(4-NITROPHENYL)-1H-BENZIMIDAZOLE SYNTHESIS

The reaction medium contained 1-(bromomethyl)-4nitrobenzene, o-phenylenediamine and pyridine- $\mathrm{N}$-oxide was heated at different temperatures and time intervals. The amount of 2-(4-nitrophenyl)-1H-benzimidazole depends on temperature, time and molar ratio of reactants (table 1). Optimal parameters of 2-(4-nitrophenyl)-1Hbenzimidazole synthesis correspond to a temperature of $110{ }^{\circ} \mathrm{C}$, two hours of reaction time and molar ratio 1(bromomethyl)-4-nitrobenzene : o-phenylenediamine : pyridine- $N$-oxide $=1: 2.5: 1$ (tablel, entry 4, yield $90 \%$ ).

We think that 1-(bromomethyl)-4-nitrobenzene is oxidized to 4-nitrobenzaldehyde (I) with the help of pyridine- $N$-oxide. This reaction looks like Kornblum reaction. 4-Nitrobenzaldehyde reacts with 0 phenylenediamine to form the Schiff base, 2-( (4nitrobenzylidene)amino)aniline (II). An intramolecular cycloaddition of Schiff base produces 2-(4-nitrophenyl)2,3-dihydro- $1 \mathrm{H}$ - benzimidazole (III). Furthermore, the oxidation of 2-(4-nitrophenyl)-2,3-dihydro- $1 \mathrm{H}$ - benzimidazole by pyridine- $\mathrm{N}$-oxide gives 2-(4-nitrophenyl)- $1 \mathrm{H}$ benzimidazole (IV). The mechanism of 2- substituted benzimidazole synthesis was recently published and is depicted as bellow [38, 39]:

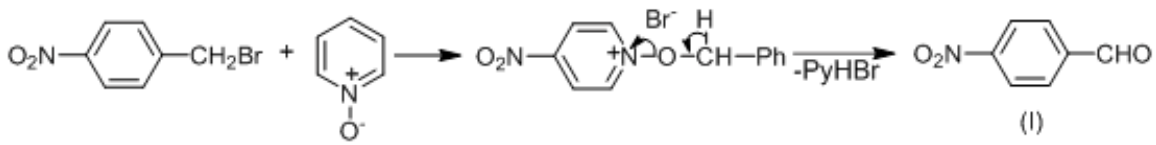

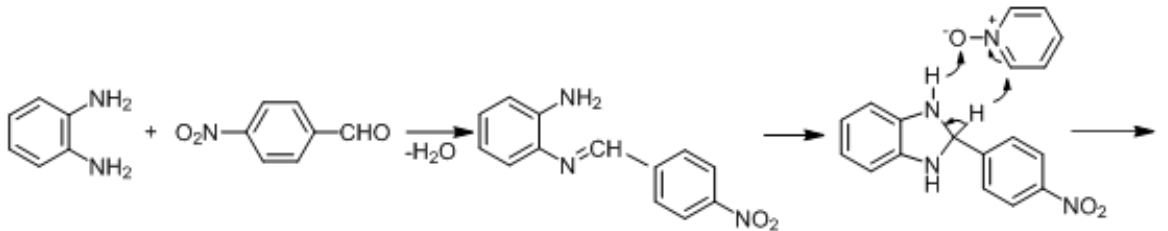

(II)

(III)<smiles>O=C[C@@H](O)c1ccncc1</smiles>

With this set of reaction conditions, a series of benzimidazoles derivatives was obtained (table 2). The synthesis of the products performs in good yields. The amount of benzimidazole derivative depends on the experimental conditions, such as the work reaction temperature, the molar ratio of reactants, the reaction time and the nature of the organic halide and amine used.

Reactions to obtain 2-substituted benzimidazoles from (bromomethyl) arenes and o-phenylenediamines occur much better than the same reactions in which alkyl primary bromides are employed. Lower boiling points of the alkyl primary bromides do not allow the development of the reactions at higher temperatures and can also be an impediment factor for the yield of synthesis of 2-alkyl substituted benzimidazoles. Reaction time is higher for 2alkyl substituted benzimidazoles than for 2-aryl substituted benzimidazoles. 
Table 2

SYNTHESIS OF 2-SUBSTITUTED BENZIMIDAZOLES UNDER SMOOTH REACTION CONDITIONS<smiles>[R][X]c1nc2ccccc2[nH]1</smiles>

\begin{tabular}{|c|c|c|c|c|c|}
\hline $\mathrm{N}^{\circ}$ & Product & $\begin{array}{l}\text { Time } \\
\text { [h] }\end{array}$ & $\begin{array}{l}\mathrm{T} \\
{\left[{ }^{\circ} \mathrm{C}\right]}\end{array}$ & $\begin{array}{l}\text { Yield } \\
{[\%]}\end{array}$ & $\begin{array}{l}\text { M.p. } \\
{\left[{ }^{\circ} \mathrm{C}\right]}\end{array}$ \\
\hline $\mathrm{I}$ & II & III & IV & V & VI \\
\hline 1 & & 2 & 105 & 80 & $\begin{array}{l}292-293[40] \\
292\end{array}$ \\
\hline $2^{--}$ & & 2 & 105 & 78 & $\begin{array}{l}269-270[41] \\
270\end{array}$ \\
\hline$\sqrt{3}$ & & 3,5 & 105 & $60^{-}$ & $\begin{array}{l}303-306[42] \\
305-306\end{array}$ \\
\hline $4^{-}$ & & 2 & $80^{-}$ & $81^{-}$ & $\begin{array}{l}203-205[43] \\
204-205\end{array}$ \\
\hline 5 & & 2 & 110 & $74^{--}$ & $\begin{array}{l}206-207[44] \\
207-208\end{array}$ \\
\hline 6 & & 6 & 35 & 30 & $\begin{array}{l}218-220[45] \\
218-219\end{array}$ \\
\hline 7 & & 2 & $110^{-}$ & 83 & $\begin{array}{l}243-244[44]^{-} \\
242-244\end{array}$ \\
\hline 8 & & 4 & $70^{-1}$ & 51 & $\begin{array}{l}173-174[46] \\
172-173\end{array}$ \\
\hline 9 & & 2 & 110 & $90^{\circ}$ & $\begin{array}{l}314-314.5[47] \\
312-314\end{array}$ \\
\hline $\mathrm{I}$ & II & III & IV & V & VI \\
\hline 10 & & 2 & 105 & 85 & $\begin{array}{l}150-151[48] \\
148-150\end{array}$ \\
\hline $11^{-}$ & & 2 & $110^{-}$ & $79^{-}$ & $\begin{array}{l}217-218[49] \\
218\end{array}$ \\
\hline 12 & & 4 & $70^{-1}$ & $75^{--}$ & $\begin{array}{l}149-153[50]^{-} \\
152-153\end{array}$ \\
\hline 16 & & 2 & 105 & 76 & $\begin{array}{l}288-289[43] \\
288-290\end{array}$ \\
\hline 13 & & 2 & 95 & 92 & $\begin{array}{l}209-210[40] \\
210\end{array}$ \\
\hline 14 & & 4 & 95 & 85 & $\begin{array}{l}191[51] \\
190-191\end{array}$ \\
\hline $15^{-}$ & & 2,5 & $100^{-}$ & 73 & $\begin{array}{l}286-287[48] \\
285-286\end{array}$ \\
\hline
\end{tabular}

\section{Conclusions}

2-Substituted benzimidazoles can be synthesized by a mild method withoutany catalyst in a solvent-free medium. The syntheses happen in dry heterogeneous medium by one potdomino reaction using o-phenylenediamines, alkyl bromides or (bromomethyl) arenes and pyridine- $\mathrm{N}$-oxide. Operational simplicity, easy work methodology and good yields are the main advantages. The method is a good alternative to protocols that use expensive catalysts, toxic solvents, and strong acids.

\section{References}

1. NARASIMHAN, B., SHARMA, D., KUMAR, P., Med. Chem. Res., 21, 2012, p. 269.

2. FLORES-CARRILLO, P., VELAZQUEZ-LOPEZ, J. M., AGUAYO-ORTIZ, R., HERNANDEZ-CAMPOS, A., TREJ O-SOTO, P. J., YEPEZ-MULIA, L., CASTILLO, R., Eur. J. Med. Chem., 137, 2017, p. 211.
3. EL-GOHARY, N. S., SHAABAN, M. I., Eur. J. Med. Chem., 137, 2017, p. 439.

4. SALAHUDDIN SHAHARYAR, M., MAZUMDER, A., ABDULLAH, M. M., Arab. J. Chem., 10, 2017, p.503.

5. BOUCHOUIT, M., SAID, M. E., ALI, M. K., BOUACIDA, S., MERAZIG, H., CHAOUCHE, N. K., CHIBANI, A., ZOUCHOUNE, B., BELFAITAH, A., BOURAIOU, A., Polyhedron, 119, 2016, p.248

6. WEN, J., LUO, Y. L., ZHANG, H. Z., ZHAO, H. H., ZHOU, C. H., CAI, G. X., Chin. Chem. Lett, 27, 2016, p. 391.

7. TONELLI, M.; NOVELLI, F., TASSO, B., VAZZANA, I., SPARATORE, A., BOIDO, V., SPARATORE, F., LA COLLA, P., SANNA, G., GILIBERTI, G., BUSONERA, B., FARCI, P., IBBA, C., LODDO, R., Bioorganic Med. Chem., 22, 2014, p. 4893.

8. VAUSSELIN, T., SERON, K., LAVIE, M., MESALAM, A. A., LEMASSON, M., BELOUZARD, S., FENEANT, L., DANNEELS, A., ROUILLE, Y., COCQUEREL, L., FOQUET, L., ROSENBERG, A. R., WYCHOWSKI, C., 
MEULEMAN, P., MELNYK, P., DUBUISSON, J., J. Virol., 90, 2016, p. 8422.

9. LIU, S. H., NELSON, C. A., XIAO, L., LU, L., SETH, P. P., DAVIS, D. R., HAGEDORN, C. H., Antivir. Res., 89, 2011, p. 54.

10. SHARMA, R., BALI, A., CHAUDHARI, B. B., Bioorganic Med. Chem. Lett., 27, 2017, p. 3007.

11. SONDHI, S. M., MAGAN, A., MAHESH, V. K., SRIMAL, R. C., GOEL, A. K., Indian. J. Chem. B, 33, 1994, p. 1144.

12. SIMON, A., BESANCON, M., SHIN, J. M., SACHS, G., Gastroenterology, 104, 1993, p. A192

13. SHRIVASTAVA, N., NAIM, M. J., ALAM, M. . ., NAWAZ, F., AHMED, S., ALAM, O., Arch. Pharm. (Weinheim), 350, 2017, p. 1.

14. USTUN, E., OZGUR, A., COSKUN, K. A., DUSUNCELI, S. D., OZDEMIR, I., TUTAR, Y., Transit. Metal Chem., 42, 2017, p. 331.

15. PROSSER, K. E., CHANG, S. W., SARACI, F. LE, P. H., WALSBY, C. J., J. Inorg. Biochem., 167, 2017, p. 89.

16. KALINOWSKA-LIS, U., SZABLOWSKA-GADOMSKA, I., LISOWSKA, K., OCHOCKI, J., MALECKI, M., FELCZAK, A., Z Anorg. Allg. Chem., 643, 2017, p. 993

17. BHATTACHARYYA, S., PURKAIT, K., MUKHERJ EE, A., Dalton Trans., 46, 2017, p. 8539.

18. ZHAO, J. A., YU, H. B., ZHI, S. C., MAO, R. N., HU, J. Y., WANG, X. X., Chin. Chem. Lett., 28, 2017, p. 1539.

19. MITRA, I., REDDY, B. V. P., MUKHERJ EE, S., LINERT, W., MOI, S. C., Chem. Phys. Lett., 678, 2017, p. 250.

20. HOEBRECKER, F., Ber., 5, 1872, p. 920.

21. WRIGHT, J. B., Chem. Rev., 48, 1951, p .397.

22. WAGNER, E. C., MILLETT, W. H., Org. Synth. Coll. Vol., 2, 1943, p. 65.

23. REFAAT, H. M., Eur. J. Med. Chem., 45, 2010, p. 2949.

24. MARIAPPAN, G., HAZARIKA, R., ALAM F., KARKI, R., PATANGIA, U., NATH, S., Arab. J. Chem., 8, 2015, p. 715.

25. MAHESH, D., SADHU, P., PUNNIYAMURTHY, T., J. Org. Chem., 80, 2015, p. 1644.

26. BAHRAMI, K., KHODAEI, M. M., NAALI, F., J. Org. Chem., 73, 2008, p. 6835.

27. BAHRAMI K, KHODAEI MM, KAVIANINIA, I., Synthesis, 4, 2007, p.417.

28. RATHOD, C.P., RAJURKAR, R.M., THONTE, S.S., Indo Am. J. Pharm. Res., 3, 2013, p. 2323.
29. EVINDAR, G., BATEY, R.A., J. Org. Chem., 71, 2006, p. 1802. 30. YANG, D., FU, H., HU, L., JIANG, Y., ZHAO, Y., J. Org. Chem., 73, 2008, p. 7841.

31. SHARMA, A.K., JOSHI, H., BHASKAR, R., SINGH, A. K., Dalton Trans., 46, 2017, p. 2228.

32. MAJUMDAR, S., CHAKRABORTY, A., BHATTACHARJEE, S., DEBNATH, S., MAITI, D. K., Tetrahedron Lett., 57, 2016, p. 4595.

33. YANG, D., FU, H., HU, L., JIANG, Y. AND ZHAO, Y., J. Org. Chem., 73, 2008, p. 7841.

34. KARHALE, S., PATIL, K., BHENKI, C., RASHINKAR, G., HELAVI, V., Res. Chem. Intermed., 42, 2016, p. 7257.

35. SHINTRE, S. A., RAMJ UGERNATH, D., SINGH, P., MOCKTAR, C., KOORBANALLY, N. A., Med. Chem. Res., 26, 2017, p. 484.

36. VAIDYANATHAN, S; SURBER, B.W., Tetrahedron Lett., 46, 2005, p. 5195.

37. SAPKAL, B. M., LABHANE, P. K., SATAM, J. R., Res. Chem. Intermed., 43, 2017, p. 4967.

38. BRATULESCU, G., Synth. Commun., 47, 2017, p. 811.

39. BRATULESCU, G, ARKIVOC, IV, 2017, p. 95.

40. ALLOUM, A. B., BOUGRIN, K., SOUFIAOUI, M., Tetrahedron Lett., 44, 2003, p. 5935.

41. PROKOPCOVA, H., KAPPE, C. O., J. Org. Chem., 72, 2007, p. 4440. 42. KIM, Y., KUMAR, M. R., PARK, N., HEO, Y., LEE, S., J. Org. Chem., 76, 2011, 76, p. 9577.

43. PONNALA, S., SAHU, D. P., Synth. Commun., 36, 2006, p. 2189.

44. SAHA, D., SAHA, A., RANU, B. C., Green Chem., 11, 2009, p. 733.

45. DAOSHAN, Y., HUA, F., LIMING, H., YUYANG, J., YUFEN, Z., J. Org. Chem., 73, 2008, p. 7841.

46. JING, X., ZHU, Q., XU, F., REN, X., LI, D., YAN, C., Synth. Commun., 36, 2006, 2597.

47. SHEN, M. G., CAl, C., J. Fluorine Chem., 128, 2007, p. 232.

48. CHEN, CH., CHEN, C., LI, B., TAO, J., PENG, J., Molecules, 17, 2012, p. 12506.

49. ALCALDE, E., DINARES, I., PEREZ-GARCIA, L., ROCA, T., Synthesis, 4, 1992, p. 395.

50. HUBBARD, J. W., PIEGOLS, A. M., SOEDERBERG, B.C.G., Tetrahedron, 63, 2007, p. 7077.

51. RIED, W., URLASS, G., Chem. Ber., 86, 1953, p.1101.

Manuscript received: 9.06 .2018 